\title{
PLASMA PROFILING - TIME OF FLIGHT MASS SPECTROMETRY: CONSIDERATIONS TO EXPLOIT ITS ANALYTICAL PERFORMANCE FOR MATERIALS CHARACTERIZATION
}

Rocío Muñiz, Lara Lobo*, Beatriz Fernández and Rosario Pereiro

Department of Physical and Analytical Chemistry, University of Oviedo, Julián Clavería 8, 33006 Oviedo, Spain.

* Correspondence: lobolara@uniovi.es

For the special issue: Themed issue dedicated to Prof. Alfredo Sanz-Medel 


\begin{abstract}
The combination of a pulsed glow discharge (PGD) with time-of-flight mass spectrometry (TOFMS) provides quasi-simultaneous mass spectra of fast transient signals directly from solid materials. Moreover, pulse profiles of the analytes can be useful to understand ionization and transport processes to the mass analyser. In this work, studies have been carried out for a better understanding and analytical exploitation of the capabilities of PP (Plasma Profiling)-TOFMS, the first PGD-TOFMS commercial instrument. The PGD operating conditions of the "PP-TOFMS" have been optimized and evaluated in terms of temporal signal distribution and sensitivity for a high variety of elements present in the standard reference material NIST 1262b (hot-rolled and colddrawn steel with certified composition for 24 elements). Short pulses and duty cycles have proved to be crucial for high sensitivity of heavy elements. In addition, the PPTOFMS has a blanking interface stage that allows filtering (or blanking) of up to four of the most intense ions to achieve higher sensitivities for trace and minor elements. Here, it is presented a thorough investigation to understand the blanking behaviour and its benefits. Results have shown that sensitivity increases for high $\mathrm{m} / \mathrm{z}$ isotopes when attenuating the major ion coming from the discharge gas $\left({ }^{40} \mathrm{Ar}\right)$ and from the major matrix isotope $\left({ }^{56} \mathrm{Fe}\right)$ : e.g. sensitivity increases up to a factor of 100 times in case of $\mathrm{W}$ as compared to no blanked experiments.
\end{abstract}

Keywords: Pulsed glow discharge; Time of flight mass spectrometry; Direct solid elemental analysis; Pulse profile. 


\section{Introduction}

A huge number of applications have been described using glow discharge (GD) coupled either to optical emission spectrometry (OES) or to mass spectrometry (MS) for direct solid analysis of varied materials. ${ }^{1-5}$ Moreover, extensive experimental and modelling studies have been carried out with GDs in order to improve and understand fundamental aspects of this atom and ion source. ${ }^{6,7}$ Though GD spectroscopies can be considered well established analytical techniques, instrumental and method developments are still in growth. Some of the implementations include the application of pulsed GDs (PGDs) in the millisecond or microsecond regime coupled to OES or MS, ${ }^{8,9}$ and the use of time of flight (TOF) as GD mass analyser. ${ }^{10}$

During the last years, PGD-TOFMS has been intensively investigated and interesting elemental and molecular analytical examples can be found in the literature. ${ }^{11,12}$ The possibilities of quasi-simultaneous multielemental detection together with fast acquisition rates make this configuration ideal for depth profile analysis of nanometric and micrometric thin layers. In addition, its software allows recording, for every data point in the analysis, each corresponding pulse profile and mass spectra so that there is plenty of information available for evaluation.

The "Plasma Profiling Spectrometer (PP-TOFMS)" from Horiba Scientific (France) is the first commercial instrument based on a radiofrequency (rf) PGD source coupled to a TOFMS. ${ }^{13,14}$ Final instrumental design of PP-TOFMS is based on two previous prototypes, ${ }^{15,16}$ being the commercial version characterized by a blanking interface stage located after the skimmer cone, horizontal sample loading and the possibility to detect negative ions. ${ }^{17}$ The blanking interface has been implemented with the purpose of filtering most intense ions within the plasma (e.g. ${ }^{40} \mathrm{Ar}^{+}$and matrix ions) so that higher sensitivity, reduced detector saturation and increased dynamic range could be achieved for the trace and minor elements in the sample. With this instrument, filtering or blanking can be applied up to four different ions simultaneously. Little information can be found in the literature regarding this tool. ${ }^{18}$

Within this context, basic studies are required to get the full benefit of PP-TOFMS and to exploit its capabilities for analytical applications in materials characterization. Such purpose was first undertaken by evaluating the effect of the PGD parameters (pressure, applied power, pulse width and pulse period) on analytical sensitivity. As model sample, a steel material was chosen. Attention will be also paid to the ion 
intensities dependence along the pulse profile region as a function of the PGD operating conditions. In addition, a systematic study of the blanking option will be discussed in order to underline the benefits and limitations of this tool in the search for analytical results with highest sensitivity.

\section{Experimental}

\subsection{Samples}

SRM NIST $1262 \mathrm{~b}$ is a hot-rolled and cold-drawn steel from National Institute of Standards and Technology was employed. This standard contains a variety of elements that expand all along the mass spectra. Concentrations of the different elements are collected in Table 1 and have been expressed all along the manuscript as mass fractions.

\subsection{Instrumentation}

"PP-TOFMS Plasma Profiling spectrometer" (Horiba Scientific, France) was used in this work. The GD ion source and the interface have been described elsewhere $[13,14]$. Briefly, the ion source is a $4 \mathrm{~mm}$ copper anode with a $20 \mathrm{~mm}$ long flow tube. The sample is placed horizontally and rf power is supplied through the back of the sample. After the interface, which is formed by a $0.5 \mathrm{~mm}$ flat sampler and a $1 \mathrm{~mm}$ conic skimmer (both cones are made of stainless steel), a blanking interface stage is placed. This blanking interface stage ${ }^{18,19}$ consists on a quadrupole acting as a mass filter for reducing unwanted ions (it can be used for up to four ions at different masses simultaneously), thus favouring enhanced sensitivity of minor elements. Basically, a rf frequency matching to a selected $\mathrm{m} / \mathrm{z}$ is superimposed to the primary confining field responsible of the ions transmission towards the TOFMS so that the ions belonging to such selected rf frequency are resonantly excited and ejected from the primary field. In practice, the user only needs to fix the undesired $\mathrm{m} / \mathrm{z}$ (to filter it) and the rf amplitude field (referred as attenuation factor along the manuscript and in the software of the instrument), which goes from 0 (no filtering) to 0.6. This tool is normally employed with matrix ions and/or major plasma gas species $\left({ }^{56} \mathrm{Fe}^{+}\right.$and ${ }^{40} \mathrm{Ar}^{+}$in the present work) whose intensity is also reduced depending on the attenuation factor chosen (typically reductions up to 3-4 orders of magnitude can be obtained). Blanking option is estimated to affect 1-2 amu units; ${ }^{19}$ however, the effect on the overall intensity may be increased at increasing the attenuation factors or when blanking more than one isotope. 
High purity Ar (99.999\% minimum purity) from Air Liquide (Oviedo, Spain) was used as discharge gas. A TOF extraction frequency of $30 \mathrm{kHz}$ was selected, obtaining a full mass spectrum (up to $290 \mathrm{~m} / \mathrm{z}$ ) every $33 \mu \mathrm{s}$. Acquisition time was fixed to obtain 1 point per second. Before each analysis, samples were flushed for $180 \mathrm{~s}$ at $300 \mathrm{~Pa}$. In all cases, the isotope of major abundance was measured except for those affected by interferences (e.g. ${ }^{117} \mathrm{Sn}^{+}$instead of ${ }^{120} \mathrm{Sn}^{+}$due to the presence of ${ }^{40} \mathrm{Ar}_{3}{ }^{+}$) or signal saturation (e.g. ${ }^{57} \mathrm{Fe}^{+}$instead of ${ }^{56} \mathrm{Fe}^{+}$). The applied power term always refers to the power applied during the pulse width. All data shown within the manuscript has been background corrected. Unless otherwise stated, experiments were carried out without blanking.

\section{Results and discussion}

\subsection{Effect of the PGD operating conditions}

First, the effect of pressure $(120-220 \mathrm{~Pa})$ and applied power $(15-90 \mathrm{~W})$ was evaluated on overall afterglow ion signals (keeping $1 \mathrm{~ms}$ pulse width and $4 \mathrm{~ms}$ period) using the conductive SRM NIST 1262b. Regarding argon pressure, Figure 1 shows that depending on the isotope, the maximum signal is obtained at slightly different pressures. For instance, ${ }^{31} \mathrm{P}^{+}$shows a continuous increase of the sensitivity up to $220 \mathrm{~Pa}$ whereas from $\mathrm{m} / \mathrm{z} 57\left({ }^{57} \mathrm{Fe}^{+}\right)$up to higher masses, the signals tend to reach a plateau at $140 \mathrm{~Pa}$ and then start to decrease for higher pressures. Previous work using a non-conductive material, has shown that the effect of pressure on signal intensity is also isotope dependent, being in all cases the maximum sensitivity found at lower Ar pressures. ${ }^{20}$ This behaviour has been also observed by other authors ${ }^{21}$ using a different PGD-TOFMS instrument; the initial increase of the intensity was attributed to maximization of Penning ionization processes, while the decrease in the afterglow was justified by collisional deexcitation of the metastable argon atom population.

Regarding ion sensitivities as a function of the applied power, sequential increases in this parameter were reflected in a slight enhancement of the signals at the assayed operational conditions: $180 \mathrm{~Pa}, 0.25 \mathrm{~ms}$ pulse, $2 \mathrm{~ms}$ period, i.e. $12.5 \%$ duty cycle (Figure 2A). However, as can be seen in Figure 2B, when higher duty cycles are employed (1 ms pulse, $4 \mathrm{~ms}$ period, i.e. $25 \%$ duty cycle), this trend is less evident and reach a plateau and/or even show a certain decrease for applied power higher than $30 \mathrm{~W}$. Even though the effective power will be lower than the applied power (a substantial fraction of the 
applied power is lost in connectors, etc.), the sputtering rate proved to be proportional to the applied power. Previous studies on the same SRM with a PGD-TOFMS prototype version showed signal increases up to $130 \mathrm{~W}$, though with a reduced slope at increasing powers. ${ }^{16}$ This behaviour was attributed be to mass load plasma effects (e.g. lower ion extraction efficiency and/or ion losses within the plasma): the higher the duty cycle the higher the mass load and so ion signals are reduced. In the commercial PP-TOFMS these effects seem to be particularly serious maybe due to the presence of the quadrupole after the skimmer in the interface; apart from mass load plasma effects, ion transmission along the quadrupole and saturation issues ${ }^{22}$ might also have an influence on the results obtained at $25 \%$ duty cycle.

Let's move now to pulse profiles. Figure 3 collects the pulse profile obtained for ${ }^{31} \mathrm{P}^{+}$, ${ }^{63} \mathrm{Cu}^{+},{ }^{90} \mathrm{Zr}^{+},{ }^{95} \mathrm{Mo}^{+}$and ${ }^{184} \mathrm{~W}^{+}$at $160 \mathrm{~Pa}, 40 \mathrm{~W}$ pulse applied power, $1 \mathrm{~ms}$ pulse width and $4 \mathrm{~ms}$ period. Similarly to the prototype instrument, ${ }^{23}$ the higher the $\mathrm{m} / \mathrm{z}$ the later the maximum of the afterglow region was obtained (a difference of about $0.13 \mathrm{~ms}$ is found between ${ }^{31} \mathrm{P}$ and ${ }^{184} \mathrm{~W}$ in our experiments). Differently to the prototype version, when using the commercial instrument it can be seen that at increasing $\mathrm{m} / \mathrm{z}$ ratios the afterglow region becomes narrower and a gap between the end of the plateau and the beginning of the afterglow is obtained. These narrower afterglows at high $\mathrm{m} / \mathrm{z}$ are reflected on the elemental sensitivity achieved by the instrument; that is, the narrower the afterglow becomes the lower sensitivity is obtained and so, the sensitivity in the high $\mathrm{m} / \mathrm{z}$ elemental region is far lower as compared to the middle $\mathrm{m} / \mathrm{z}$ region.

Aiming at further understating the pulse profiles shapes as a function of the PGD operational conditions, the influence of the pulse width and duty cycle has been also considered. The different pulse widths compared were $1 \mathrm{~ms}$ and $0.5 \mathrm{~ms}$ both using a $4 \mathrm{~ms}$ period, and $0.25 \mathrm{~ms}$ pulse width with a $2 \mathrm{~ms}$ pulse period. Figure 4 shows the afterglow pulse profiles obtained at the different conditions evaluated. As can be seen, all pulse widths tested provide analogue intensities in the afterglow at intermediate masses (e.g. ${ }^{31} \mathrm{P}^{+}$and $\left.{ }^{63} \mathrm{Cu}^{+}\right)$. Recently, Fernández et al. ${ }^{24}$ have also reported nearly no changes on the analytical sensitivity as a function of pulse width when analysing thin film tandem solar cells with PP-TOFMS. These results are also in agreement to previous work from Efimova et al. ${ }^{25}$ with PGD-OES, who observed that sensitivity hardly changed depending on the pulse width assayed. On the other hand, it is important to note that in our experiments an enhancement of the sensitivity for high mass isotopes (e.g. ${ }^{184} \mathrm{~W}^{+}$) is produced at the lowest pulse width assayed. Our hypothesis to explain such observation 
is related to the occurrence of space charge effects in the interface, which are particularly important at increasing ion populations and inversely proportional to the speed of ions: the slower ions (higher $\mathrm{m} / \mathrm{z}$ ) are more disturbed than faster ions (lower $\mathrm{m} / \mathrm{z}$ ). ${ }^{26}$ As these repulsions are therefore reduced at reduced ion populations, when short pulse widths are employed transmission of heavier ions is favoured and so an enhancement of the sensitivity is obtained.

\subsection{Evaluation of the blanking conditions on the analytical signals}

PP-TOFMS is designed with a blanking interface stage with the purpose of filtering most intense ions present in the plasma and thus, allowing the increase of analytical sensitivity for minor and trace elements and the dynamic range of the instrument. Here, the influence of the blanking conditions on the pulse profiles shapes and the analytical signals has been studied in detail. Figure 5 shows the pulse profiles obtained using two different blanking conditions (the corresponding pulse profile without any blanking is already collected in Figure 4A). In Figure 5A the filtering tool was applied to ${ }^{40} \mathrm{Ar}^{+}$, whereas in Figure 5B blanking was applied to both ${ }^{40} \mathrm{Ar}^{+}$and the major matrix element (i.e. ${ }^{56} \mathrm{Fe}^{+}$). It can be seen that blanking has an effect on both, intensities and temporal signals distribution, being the last more obvious for high $\mathrm{m} / \mathrm{z}$ ions: the higher the applied blanking, the gap between the two afterglow peaks tends to disappear. Moreover, the time difference between the second maximum in the afterglow region for ${ }^{31} \mathrm{P}^{+}$and ${ }^{184} \mathrm{~W}^{+}$becomes shorter: from about $0.13 \mathrm{~ms}$ (obtained without blanking and when blanking ${ }^{40} \mathrm{Ar}^{+}$at 0.5 attenuation factor) down to $0.10 \mathrm{~ms}$ when blanking both ${ }^{40} \mathrm{Ar}^{+}$(at 0.5 attenuation factor) and ${ }^{56} \mathrm{Fe}^{+}$(at 0.3 attenuation factor). If an attenuation factor of 0.5 is applied to both, ${ }^{40} \mathrm{Ar}^{+}$ and ${ }^{56} \mathrm{Fe}^{+}$, a difference of $0.07 \mathrm{~ms}$ is obtained. Also, higher sensitivity is obtained at increasing blanking conditions, being the enhancement more pronounced for ${ }^{184} \mathrm{~W}^{+}$. The fact that filtering most intense ions enables for similar afterglow time domains along the whole $\mathrm{m} / \mathrm{z}$ range and also similar to those previously obtained with the prototype version (without quadrupole filter) ${ }^{23}$ reflects that our previous hypothesis regarding space charge effects and/or saturation issues within the quadrupole seems reasonable.

The effect of blanking on the sensitivity has been then evaluated for 24 elements present in SRM NIST 1262b. Figure 6 collects the results obtained at the different blanking conditions assayed. As it can be seen, three regions have been distinguished along the mass spectrum: A) the region constituted by isotopes far from the blanked ions (i.e. with low or high $\mathrm{m} / \mathrm{z}$ ); B) isotopes in between the two blanked ions; C) isotopes with 
$\mathrm{m} / \mathrm{z}$ close to one of the attenuated ions. In Figure $6 \mathrm{~A}$ it can be seen that the higher the blanking the higher the sensitivity. However, in Figures $6 \mathrm{~B}$ and Figure $6 \mathrm{C}$, as the attenuation factor for ${ }^{56} \mathrm{Fe}^{+}$increases (at constant ${ }^{40} \mathrm{Ar}^{+}$) sensitivity starts to decrease. In fact, it can be observed that the closer the $\mathrm{m} / \mathrm{z}$ of the analyte to the attenuated ion, the higher the decrease in its sensitivity at increasing blanking conditions (e.g. $\mathrm{Cu}$ is less influenced by the attenuation of ${ }^{56} \mathrm{Fe}^{+}$than Co when keeping constant the ${ }^{40} \mathrm{Ar}^{+}$ attenuation factor). Regarding precision (see error bars in Figure 6) and independently of the blanking attenuation value applied in these experiments, no differences, in terms of relative standard deviations, were obtained for any of the studied isotopes as a function of the applied blanking. This blanking option turns out to be very useful when high sensitivity is required for certain applications. In this line, we have recently reported an application describing depth profile analysis of rare earth elements on corroded steels where the use of blanking turned out to be crucial to detect the sought analytes. ${ }^{14}$ Although it is out of the intended scope of the present work, it could be convenient to mention here that at a given blanking condition, linear calibration curves using different matrices are achieved.

\section{Conclusions}

In the present work, the PP-TOFMS instrument has been thoroughly investigated in order to understand its analytical performance and take advantage of the possibilities that it offers. Results showed that high mass elements $(\geq 75 \mathrm{~m} / \mathrm{z})$ show a comparatively lower sensitivity, which was attributed to the space-charge effects taking place in the interface of the instrument. In such direction, the use of shorter pulses and comparatively low duty cycles has demonstrated that contributes to improve sensitivity for such elements.

On the other hand, blanking has proved to be a valuable tool to increase sensitivity, particularly for elements with high mass. Nevertheless, careful attention needs to be paid to the selection of the blanking conditions because sensitivity can be reduced for ions with $\mathrm{m} / \mathrm{z}$ close to those attenuated. Therefore, the effect of filtering major components should be carefully evaluated in standards and samples when aiming at quantitative analysis.

\section{Conflicts of interest}

There are no conflicts to declare. 


\section{Acknowledgements}

Financial support from Horiba Scientific through a project is gratefully acknowledged. B. Fernandez acknowledges her contract RYC-2014-14985 to the Spanish Ministry of Economy and Competitiveness through the "Ramón y Cajal Program". 


\section{Legends of Figures}

Figure 1: Sensitivities (cps divided by the isotope concentration in the sample) measured over the entire afterglow time domain in SRM NIST 1262b as a function of the PGD pressure $(120 \mathrm{~Pa}-220 \mathrm{~Pa})$. Measurements were performed at $40 \mathrm{~W}$ pulse applied power, $1 \mathrm{~ms}$ pulse width and $4 \mathrm{~ms}$ period.

Figure 2: Sensitivities (cps divided by the isotope concentration in the sample) measured over the entire afterglow time domain in CSM NIST 1262b as a function of applied power. Measurements were done at: A) $0.2 \mathrm{~ms}$ pulse, $2 \mathrm{~ms}$ period and B) $1 \mathrm{~ms}$ pulse and $4 \mathrm{~ms}$ period, keeping $160 \mathrm{~Pa}$.

Figure 3: Pulse profile for ${ }^{31} \mathrm{P}^{+},{ }^{63} \mathrm{Cu}^{+}$and ${ }^{184} \mathrm{~W}^{+}$obtained at $160 \mathrm{~Pa}, 40 \mathrm{~W}$ pulse applied power, $1 \mathrm{~ms}$ pulse width and $4 \mathrm{~ms}$ period.

Figure 4: Pulse profiles for ${ }^{11} \mathrm{~B}^{+},{ }^{27} \mathrm{Al}^{+},{ }^{31} \mathrm{P}^{+},{ }^{63} \mathrm{Cu}^{+},{ }^{181} \mathrm{Ta}^{+}$and ${ }^{184} \mathrm{~W}^{+}$obtained at: A) $1 \mathrm{~ms}$ pulse, 4 ms period; B) $0.5 \mathrm{~ms}$ pulse, $4 \mathrm{~ms}$ period; C) $0.25 \mathrm{~ms}$ pulse, $2 \mathrm{~ms}$ period. All of them were performed at $180 \mathrm{~Pa}$ and $40 \mathrm{~W}$ pulse applied power. Each pulse profile shown corresponds to averaged pulse profiles obtained during the whole duration of the measurement.

Figure 5: ${ }^{31} \mathrm{P}^{+},{ }^{63} \mathrm{Cu}^{+}$and ${ }^{184} \mathrm{~W}^{+}$pulse profiles obtained when attenuating either one or two major ions with different attenuation factors: A) ${ }^{40} \mathrm{Ar}^{+}: 0.5$; B) ${ }^{40} \mathrm{Ar}^{+}: 0.5$ and ${ }^{56} \mathrm{Fe}^{+}: 0.3$. Measurements were done at $160 \mathrm{~Pa}, 40 \mathrm{~W}$ pulse applied power, 1 $\mathrm{ms}$ pulse width and $4 \mathrm{~ms}$ period. Each pulse profile shown corresponds to averaged pulse profiles obtained during the whole duration of the measurement.

Figure 6: Sensitivity (cps divided by the isotope concentration in the sample) obtained as a function of the blanking conditions $\left({ }^{40} \mathrm{Ar} 0.5 ;{ }^{40} \mathrm{Ar} 0.5,{ }^{56} \mathrm{Fe} 0.1 ;{ }^{40} \mathrm{Ar}\right.$ $\left.0.5,{ }^{56} \mathrm{Fe} 0.3 ;{ }^{40} \mathrm{Ar} 0.5,{ }^{56} \mathrm{Fe} 0.5 ;{ }^{40} \mathrm{Ar} 0.5,{ }^{56} \mathrm{Fe} 0.6\right)$. Signals shown correspond to the mean value and standard deviation of three replicate measurements obtained at $160 \mathrm{~Pa}, 40 \mathrm{~W}, 1 \mathrm{~ms}$ pulse width and $4 \mathrm{~ms}$ period. A) isotopes with $\mathrm{m} / \mathrm{z}$ far from the attenuated ions; B) isotopes with $\mathrm{m} / \mathrm{z}$ in between the two blanked ions; C) isotopes 
with $\mathrm{m} / \mathrm{z}$ close to one of the attenuated ions (the isotope collected for iron was $\left.{ }^{57} \mathrm{Fe}\right)$. 


\section{TABLES}

Table 1 Elemental concentrations $\left(\mu \mathrm{g} \cdot \mathrm{g}^{-1} \mathrm{~m} / \mathrm{m}\right)$ in SRM NIST $1262 \mathrm{~b}$. Iron is not collected because is the matrix element. Fe concentration $(95.3 \% \mathrm{~m} / \mathrm{m})$ is calculated by difference respect to the rest of the elements present in the SRM.

\begin{tabular}{llll}
\hline Element & Concentration & Element & Concentration \\
$\mathrm{B}$ & 25 & $\mathrm{Cu}$ & 5100 \\
$\mathrm{Mg}$ & 6 & $\mathrm{As}$ & 960 \\
$\mathrm{Al}$ & 810 & $\mathrm{Zr}$ & 2200 \\
$\mathrm{Si}$ & 4000 & $\mathrm{Nb}$ & 3000 \\
$\mathrm{P}$ & 440 & $\mathrm{Mo}$ & 700 \\
$\mathrm{~S}$ & 370 & $\mathrm{Ag}$ & 11 \\
$\mathrm{Ti}$ & 1000 & $\mathrm{Sn}$ & 160 \\
$\mathrm{~V}$ & 410 & $\mathrm{Sb}$ & 120 \\
$\mathrm{Cr}$ & 3000 & $\mathrm{Ta}$ & 2000 \\
$\mathrm{Mn}$ & 10500 & $\mathrm{~W}$ & 2000 \\
$\mathrm{Ni}$ & 5900 & $\mathrm{~Pb}$ & 4 \\
$\mathrm{Co}$ & 3000 & & \\
\hline
\end{tabular}




\section{References}

1 L. Lobo, B. Fernández and R. Pereiro, "Depth profile analysis with glow discharge spectrometry", J. Anal. At. Spectrom., 2017, 32, 920-930.

2 S. Mohajernia, A. Mazare, I. Hwang, S. Gaiaschi, P. Chapon, H. Hildebrand and P. Schmuk, "Depth elemental characterization of 1D self-aligned TiO2 nanotubes using calibrated radio frequency glow discharge optical emission spectroscopy (GDOES)", Appl. Surf. Sci., 2018, 442, 412-416.

3 Z. Weiss and P. Vlcak, "Analysis of shallow depth profiles of titanium nitride and Nimplanted titanium by GD-OES: the 'hydrogen effect' after the discharge startup and a correction thereof", J. Anal. At. Spectrom., 2017, 32, 2476-2484.

4 M. Di Sabatino, A. L. Dons, J. Hinrichs and L. Arnberg, "Determination of relative sensitivity factors for trace element analysis of solar cell silicon by fast-flow glow discharge mass spectrometry", Spectrochim. Acta B, 2011, 66, 144-148.

5 M. Bouza, L. Lobo, R. Muñiz, R. Pereiro, J. Esparza and J. A. García, "Characterization of gold coated ceramics by radiofrequency pulsed glow discharge - time of flight mass spectrometry", J. Anal. At. Spectrom., 2018, 33, 502-507.

6 Z. Weiss, E. B. M. Steers and J. C. Pickering, "Transition rate diagrams and excitation of titanium in a glow discharge in argon and neon", Spectrochim. Acta B, 2018, 144, $20-28$.

7 A. Bogaerts, "The afterglow mystery of pulsed glow discharges and the role of dissociative electron-ion recombination”, J. Anal. At. Spectrom., 2007, 22, 502-512.

$8 \mathrm{Ph}$. Belenguer, M. Ganciu, Ph. Guillot and Th. Nelis, "Pulsed glow discharges for analytical applications", Spectrochim. Acta B, 2009, 64, 623-641.

9 M. Voronov and V. Hoffmann, "Application of microsecond pulsed glow discharge to modern commercially available optical emission spectrometers for bulk elemental analysis", J. Anal. At. Spectrom., 2018, 33, 663-669.

10 M. Hohl, A. Kanzari, J. Michler, T. Nelis, K. Fuhrer and M. Gonin, "Pulsed r.f.-glowdischarge time-of-flight mass spectrometry for fast surface and interface analysis of conductive and non-conductive materials", Surf. Interface Anal., 2006, 38, 292-295.

11 R. Pereiro, A. Solà-Vázquez, L. Lobo, J. Pisonero, N. Bordel, J. M. Costa and A. SanzMedel, "Present and future of glow discharge - Time of flight mass spectrometry in analytical chemistry", Spectrochim. Acta B, 2011, 66, 399-412. 
12 J. Pisonero, N. Bordel, C. Gonzalez de Vega, B. Fernández, R. Pereiro and A. SanzMedel, "Critical evaluation of the potential of radiofrequency pulsed glow dischargetime-of-flight mass spectrometry for depth-profile analysis of innovative materials", Anal. Bioanal. Chem., 2013, 405, 5655-5662.

13 R. Muñiz, L. Lobo, K. Németh, L. Péter and R. Pereiro, "Evaluation of different strategies for quantitative depth profile analysis of $\mathrm{Cu} / \mathrm{NiCu}$ layers and multilayers via pulsed glow discharge - time of flight mass spectrometry”, Spectrochim. Acta B, 2017, 135, 34-41.

14 R. Muñiz, L. Lobo, T. Kerry, C. A. Sharrad and R. Pereiro, "Depth profile analysis of rare earth elements in corroded steels by pulsed glow discharge - time of flight mass spectrometry", J. Anal. At. Spectrom., 2017, 32, 1306-1311.

15 S. Canulescu, I. S. Molchan, C. Tauziede, A. Tempez, J. A. Whitby, G. E. Thompson, P. Skeldon, P. Chapon and J. Michler, "Detection of negative ions in glow discharge mass spectrometry for analysis of solid specimens", Anal. Bioanal. Chem., 2010, 396, 2871-2879.

16 L. Lobo, J. Pisonero, N. Bordel, R. Pereiro, A. Tempez, P. Chapon, J. Michler, M. Hohl and A. Sanz-Medel, "A comparison of non-pulsed radiofrequency and pulsed radiofrequency glow discharge orthogonal time-of-flight mass spectrometry for analytical purposes", J. Anal. At. Spectrom., 2009, 24, 1373-1381.

17 L. Lobo, B. Fernández, R. Muñiz, R. Pereiro and A. Sanz-Medel, "Capabilities of radiofrequency pulsed glow discharge-time of flight mass spectrometry for molecular screening in polymeric materials: positive versus negative ion mode", J. Anal. At. Spectrom., 2016, 31, 212-219.

18 US Patent 2008/0149825.

19 EP 1968100 A1.

20 M. Bouza, L. Lobo, R. Muñiz, R. Pereiro, J. Esparza and J. A. García, "Characterization of gold coated ceramics by radiofrequency pulsed glow discharge time of flight mass spectrometry", J. Anal. At. Spectrom., 2018, 33, 502-507.

21 M. R. DeJesus, G. Gu, F. L. King, J. H. Barnes and C. L. Lewis, "Ion formation in millisecond pulsed glow discharge plasmas”, J. Anal. At. Spectrom., 2011, 26, 22062215.

22 P. H. Dawson, Quadrupole Mass Spectrometry and its applications, Elsevier Scientific Publishing Company, Amsterdam, 1976. 
23 M. Bouza, B. Fernández, R. Pereiro, N. Bordel and A. Sanz-Medel, "Pulsed radiofrequency glow discharge time of flight mass spectrometry for coated glass analysis", J. Anal. At. Spectrom., 2015, 30, 1108-1116.

24 B. Fernández, L. Lobo, N. Reininghaus, R. Pereiro and A. Sanz-Medel, "Characterization of thin film tandem solar cells by radiofrequency pulsed glow discharge - time of flight mass spectrometry", Talanta, 2017, 165, 289-296.

25 V. Efimova, V. Hoffmann and J. Eckert, "Sputter crater formation in the case of microsecond pulsed glow discharge in a Grimm-type source. Comparison of direct current and radio frequency modes", Spectrochim. Acta B, 2012, 76, 181-189.

26 K. Busch, "Space charge in mass spectrometry”, Spectroscopy, 2004, 19, 35-38. 


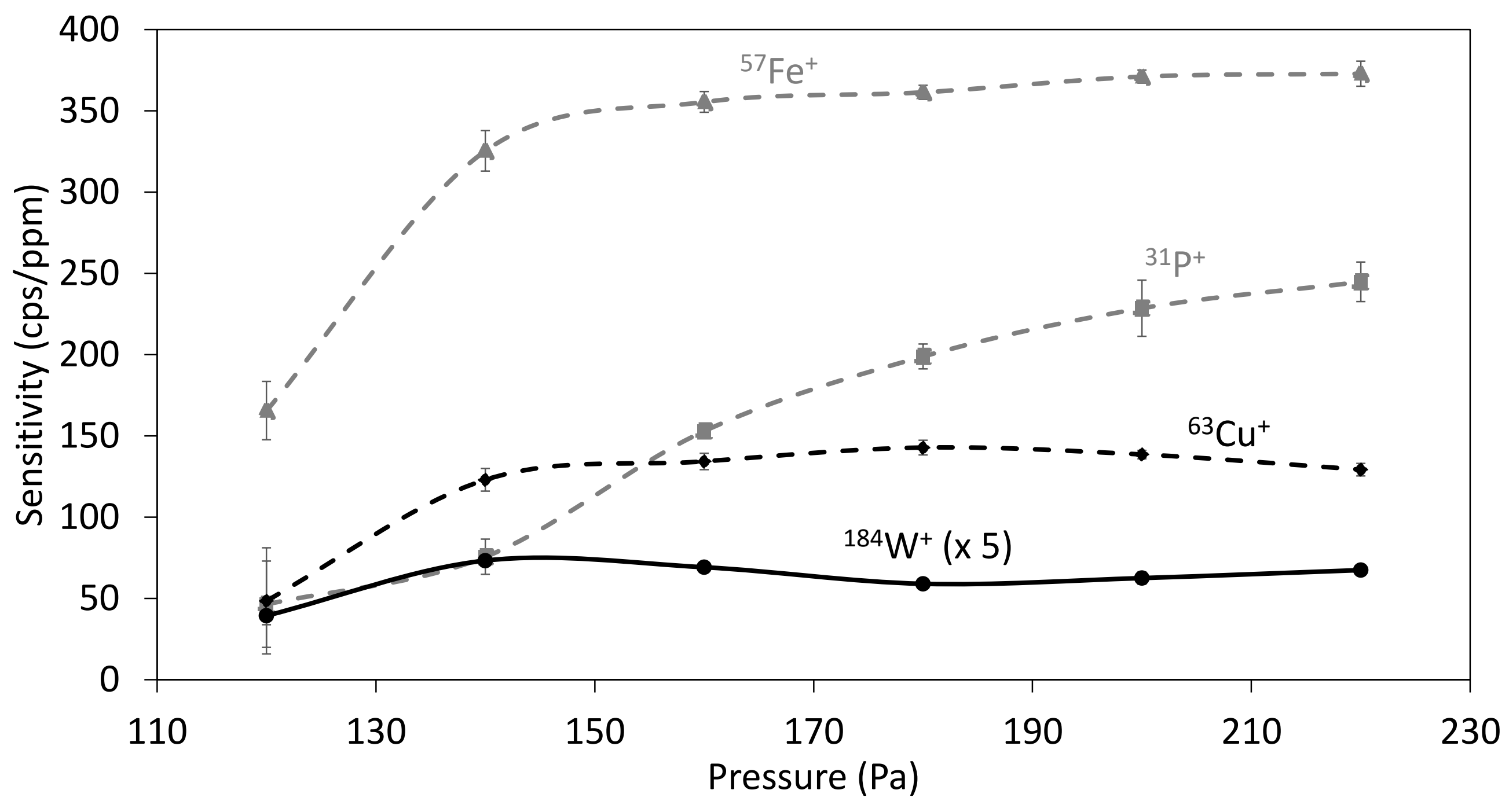

Figure 1 

A) $12.5 \% \mathrm{DC}$

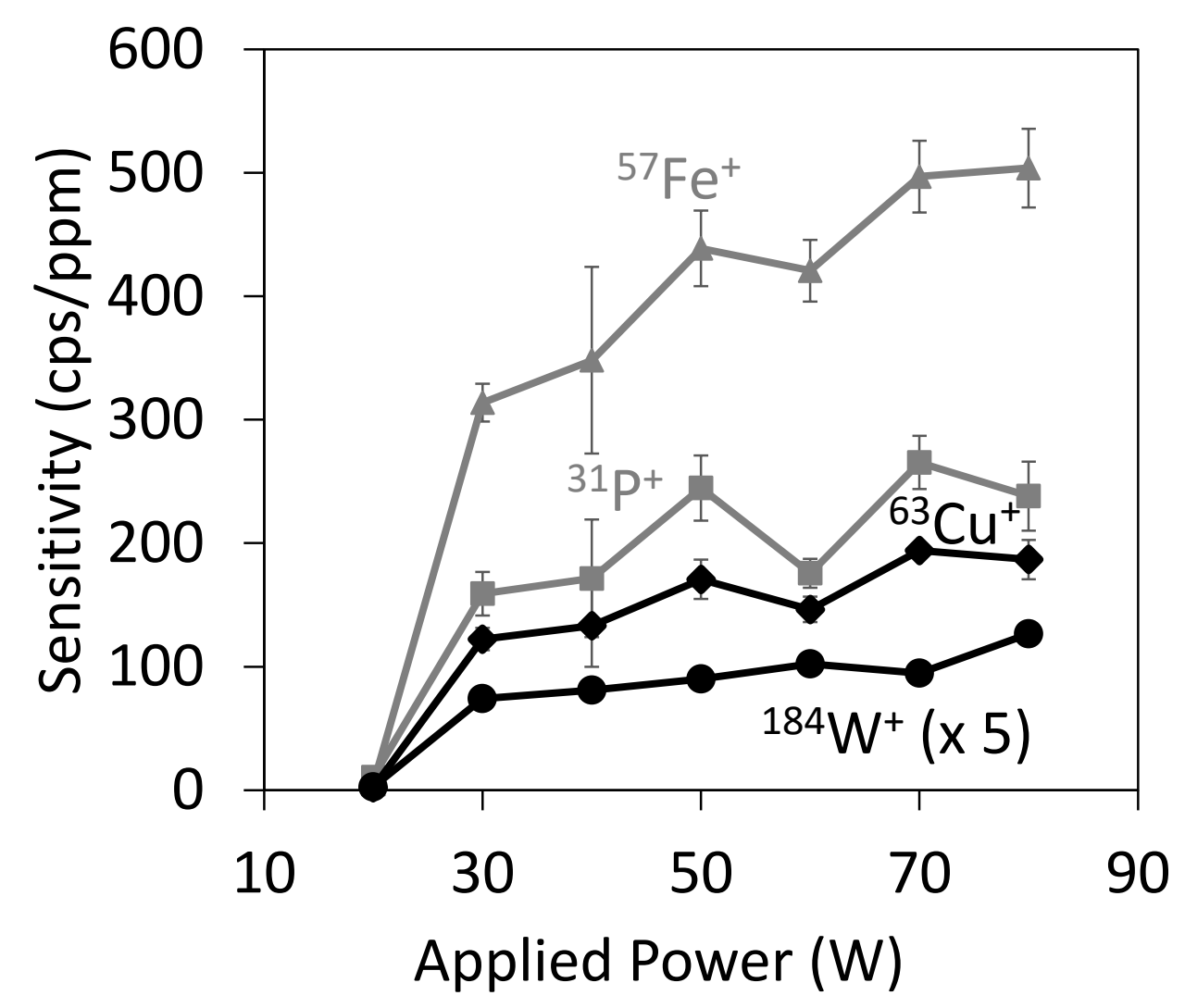

B) $25 \% \mathrm{DC}$

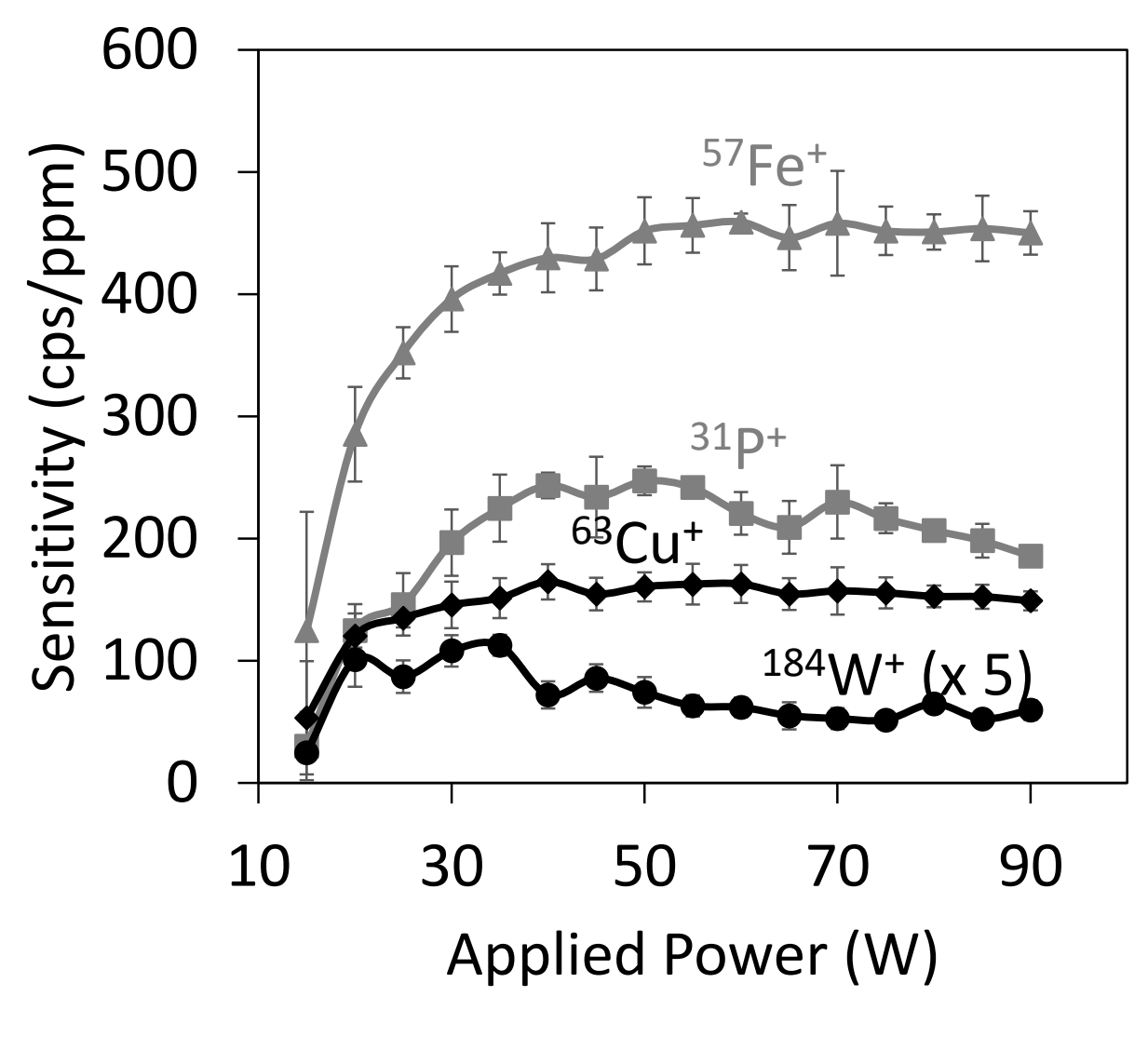




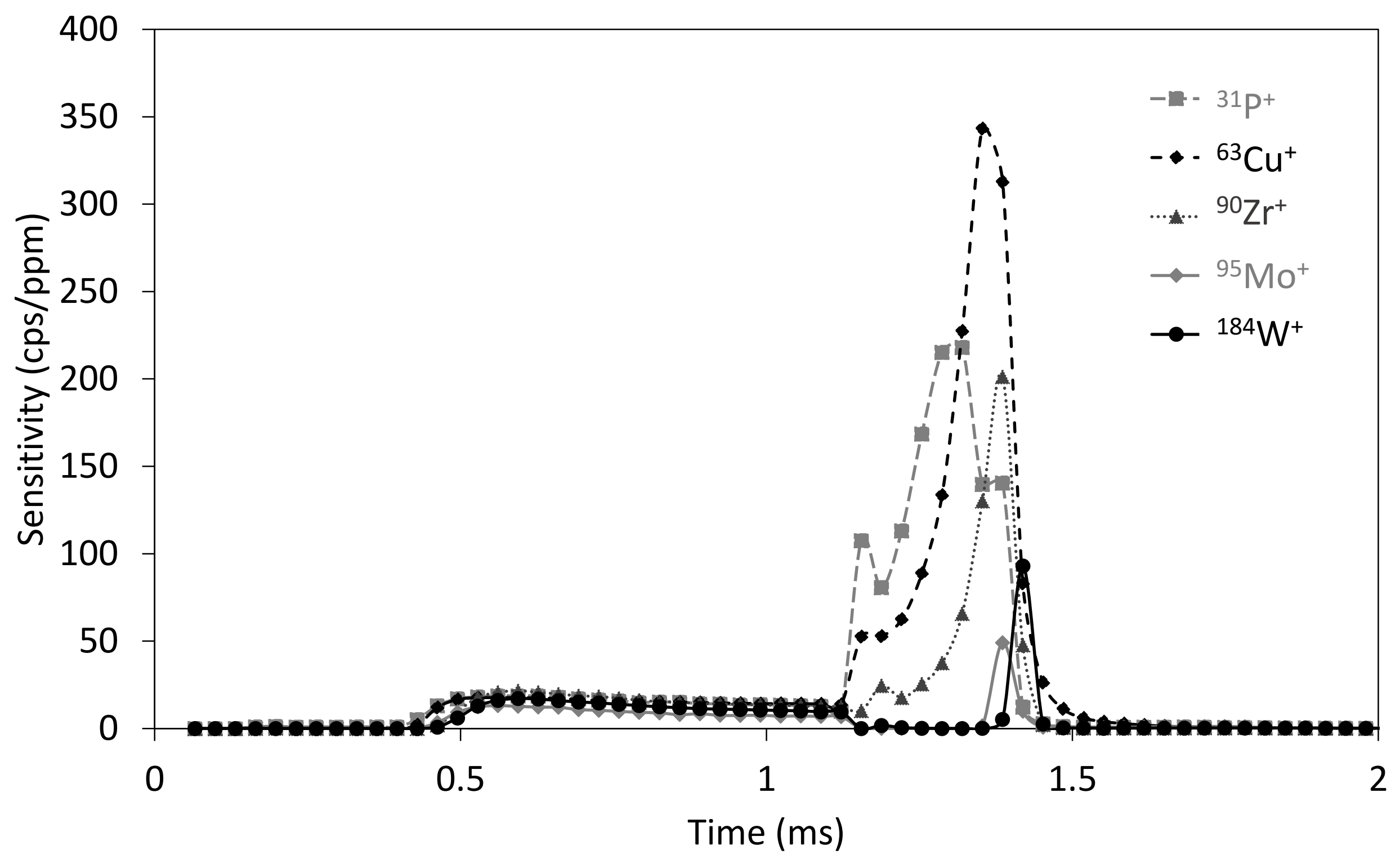

Figure 3 
A) $1 \mathrm{~ms}$ pulse/4 ms period

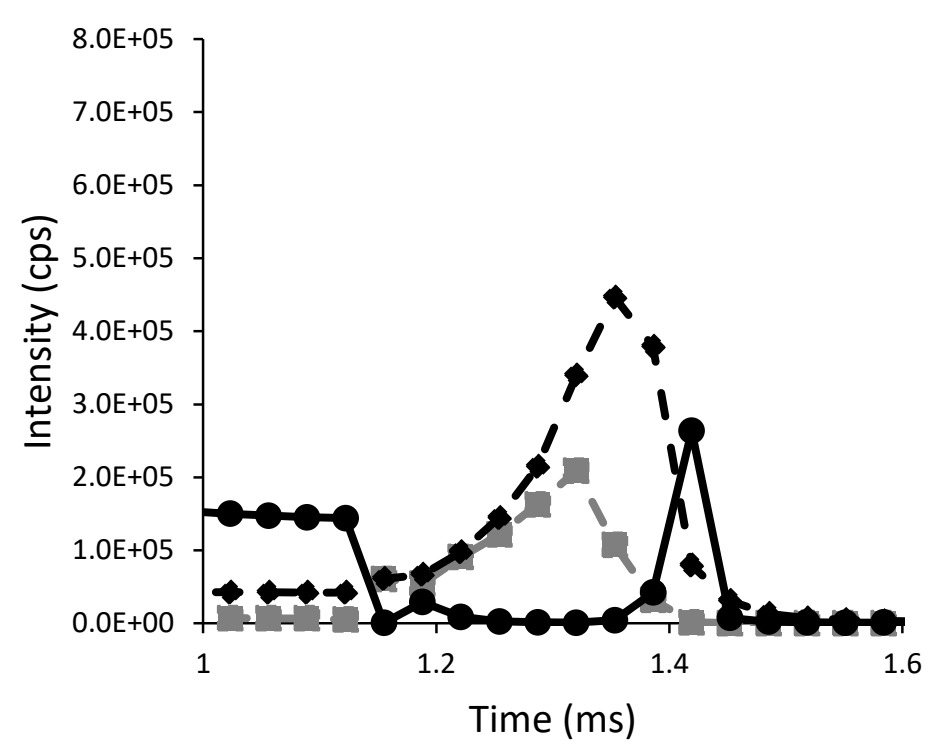

B) $0.5 \mathrm{~ms}$ pulse/4 ms period

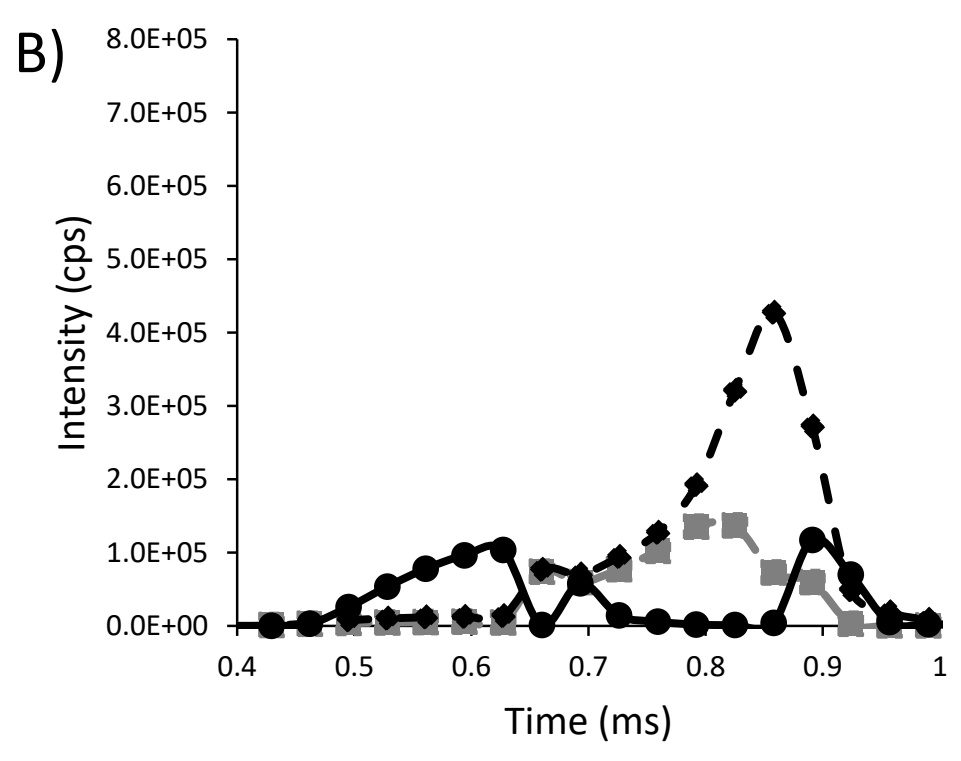

C) $0.25 \mathrm{~ms}$ pulse $/ 2 \mathrm{~ms}$ period

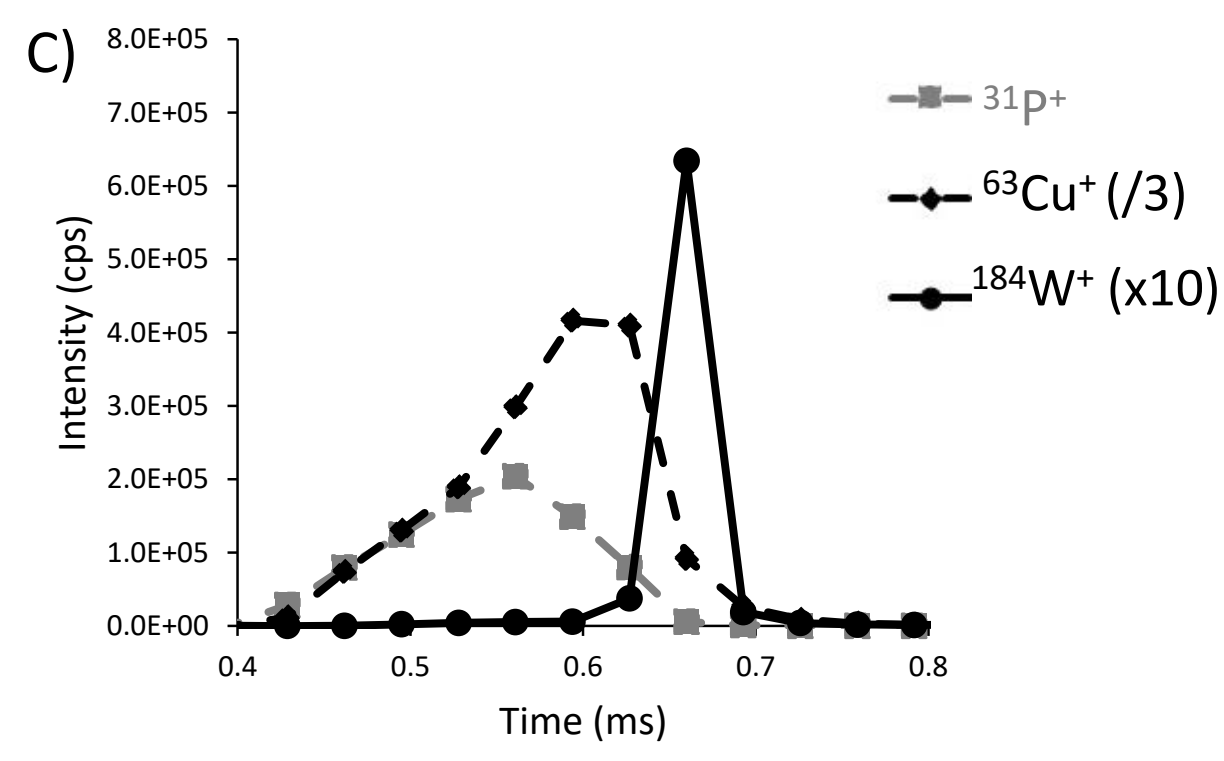


A)

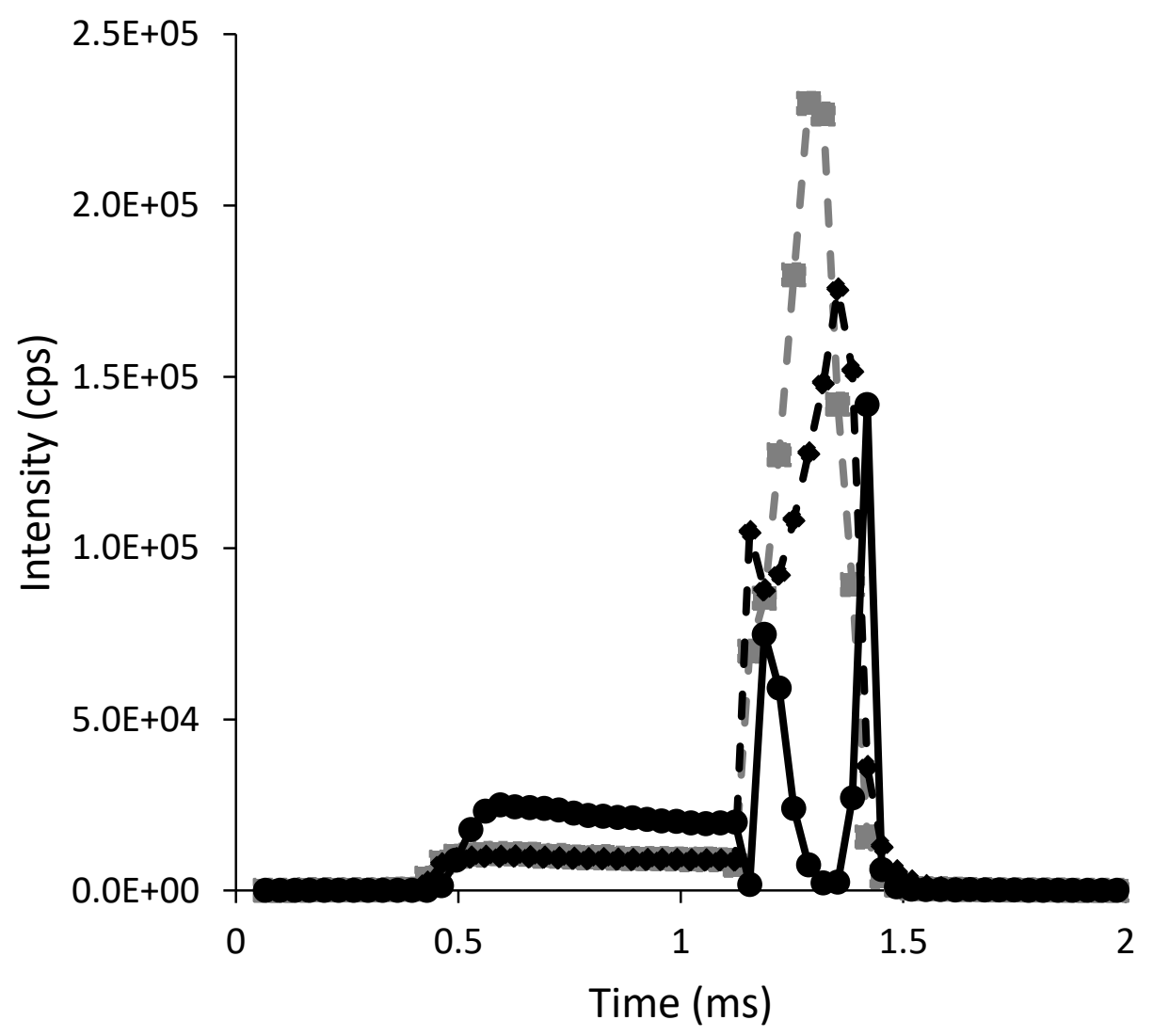

B)

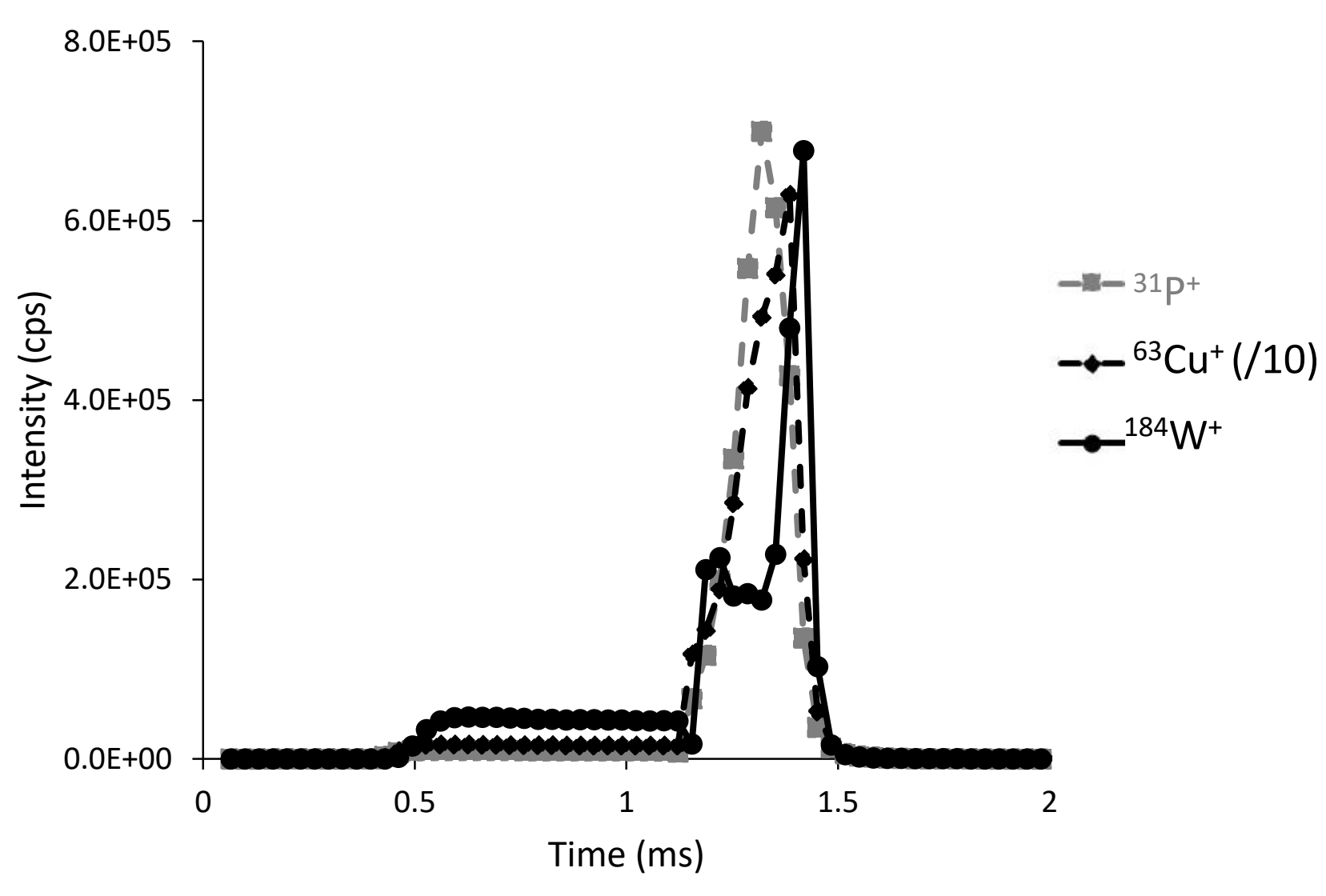

Figure 5 
A)

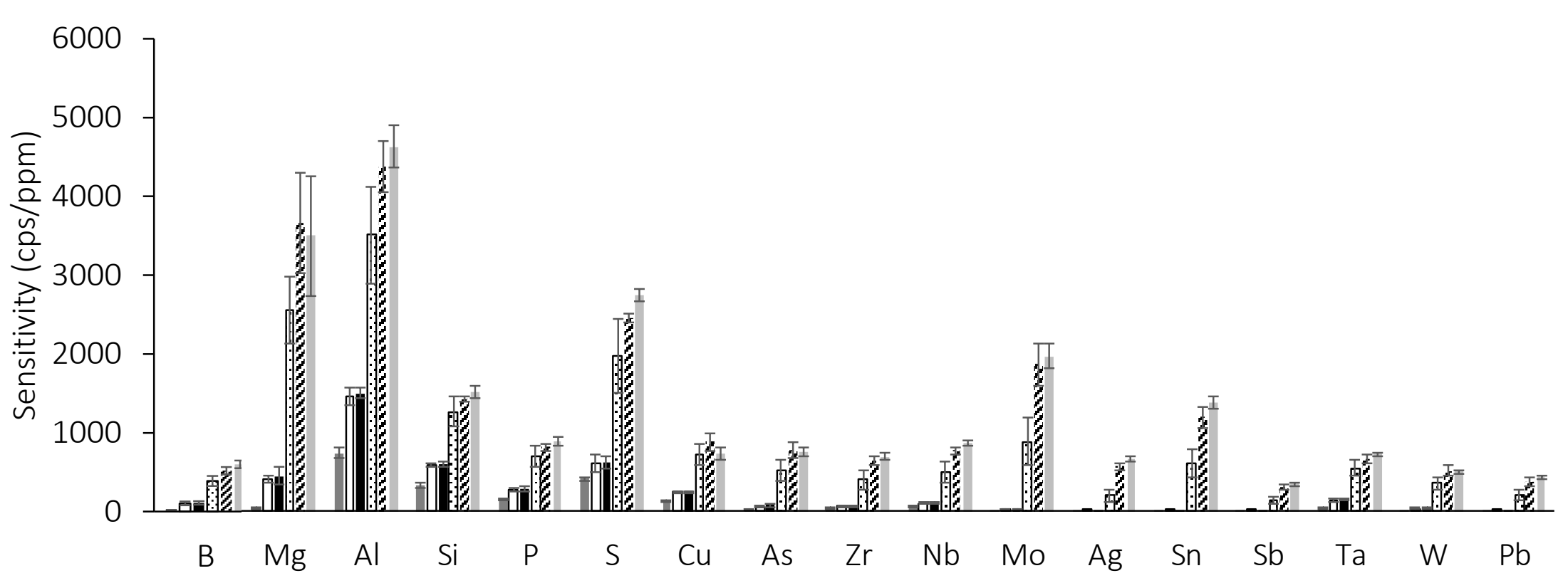

Without blanking

$\square \operatorname{Ar} 0.5$

- Ar 0.5 Fe 0.1

궁 0.5 Fe 0.3

/ Ar 0.5 Fe 0.5

Ar 0.5 Fe 0.6

B)

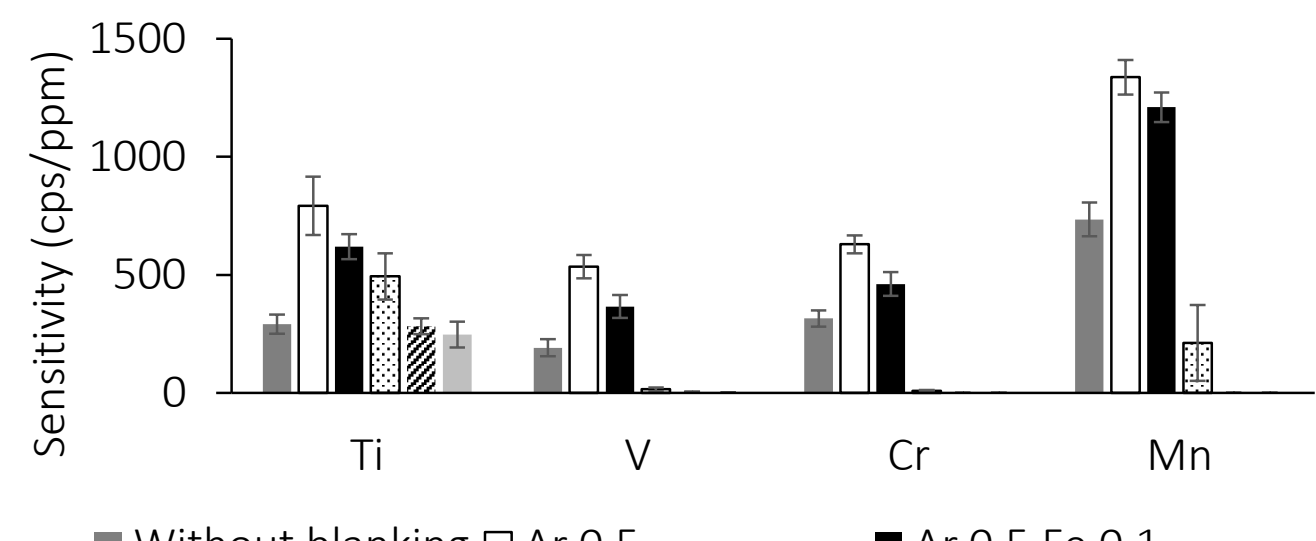

- Without blanking $\square \operatorname{Ar} 0.5$

圆Ar $0.5 \mathrm{Fe} 0.3 \quad \% \operatorname{Ar} 0.5 \mathrm{Fe} 0.5$
- Ar $0.5 \mathrm{Fe} 0.1$

Ar $0.5 \mathrm{Fe} 0.6$
C)

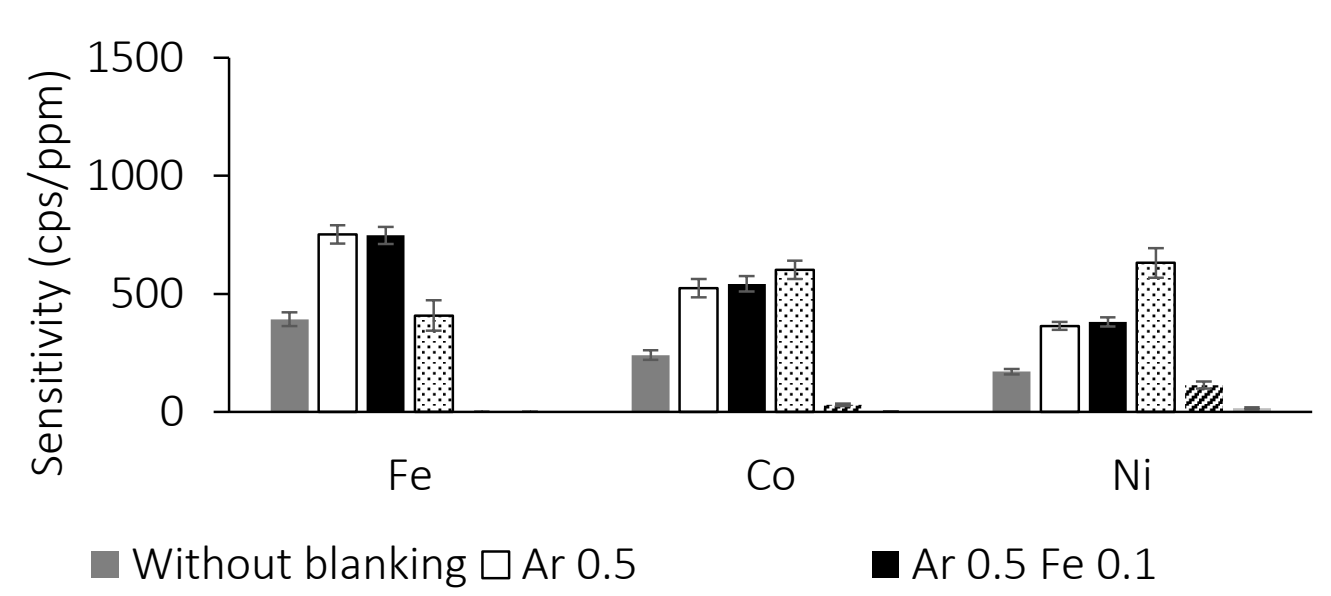

圈 $\mathrm{Ar} 0.5 \mathrm{Fe} 0.3 \quad$ \# Ar $0.5 \mathrm{Fe} 0.5$ 